\title{
Criação de um Web Site para enfermeiros sobre Pé Diabético*
}

\author{
Creation of Web Site for nurses about Diabetic Foot \\ Creación de un Sitio Web para los enfermeros sobre el pie diabético \\ Vera Lúcia Souza Alves ${ }^{1}$ Isabel Cristina Kowal Olm Cunha², \\ Heimar F. Marin ${ }^{3}$, Odete de Oliveira ${ }^{4}$
}

\section{RESUMO}

Objetivos: Descrever o processo de criação de um web site educacional para enfermeiros contendo informações sobre avaliação e cuidados preventivos dos pés em pacientes com diabetes mellitus. Métodos: Pesquisa aplicada desenvolvida em 2004, baseada no modelo teórico de Tronchim (1999) para a construção de web sites, composto das fases de conceitualização, desenvolvimento, implementação e avaliação. Resultados: O site possui 68 páginas contendo informações, orientações, fotos e ilustrações, estruturadas em tópicos com os conceitos básicos do diabetes mellitus, pé diabético, neuropatias, doença vascular periférica, infecções, amputações, educação e orientações de enfermagem. Foi avaliado por especialistas que referendaram seu conteúdo e fizeram sugestões para seu aperfeiçoamento.Conclusões: A criação e desenvolvimento do site foram descritos, podendo ser acessado no www.unifesp.br/denf/nien na opção pesquisa.

Descritores: Enfermagem; Educação à distância; Pé diabético.

\begin{abstract}
Aim: This product consists in describe the creation process of an educational web site displaying information on evaluation and preventive care of feet in patients with diabetes mellitus also to evaluate the information content available in the web site with professionals. Methods: It is an applied research based on the Tronchim Theoretical Model (1999) for the web site construction. Results: The site has 68 pages with basic concepts of diabetes mellitus, diabetic foot, neuropathies, peripheral vascular disease, infections, amputations, education and nursing orientations. Conclusion: The site can be accessed by the URL: www.unifesp.br/denf/nien, in the option research.
\end{abstract}

Keywords: Nursing; Education, distance; Diabetic foot.

\section{RESUMEN}

Objetivos: Neste estudio los objetivos fueron desarrollar el proceso de creación de un web site educacional com informaciones sobre la evaluación y los cuidados preventivos de los pies en pacientes con diabetes mellitus y evaluar el contenido de las informaciones disponibilizadas en el web site con especialistas. Métodos: Trátase de una pesquisa aplicada baseada en el modelo te'orico de Tronchim (1999), para la construcción de un web site. Resultados: El site posee 68 páginas, con conceptos básicos de diabetes mellitus, pie diabético, neuropatías, enfermedad vascular periférica, infecciones, amputaciones, educación y orientaciones de enfermería. Conclusion: El site puede ser accesado a través de la URL: www.unifesp.br/denf/nien, en la opición pesquisa.

Descriptores: Enfermería; Educación a distancia; Pie diabético.

\footnotetext{
* Parte da Dissertação de Mestrado, apresentada à Universidade Federal de São Paulo - UNIFESP - São Paulo (SP) - Brasil.

${ }^{1}$ Enfermeira do Hospital Beneficência Portuguesa de São Paulo - São Paulo (SP) - Brasil.

${ }^{2}$ Doutora em Enfermagem. Professora Adjunta da Universidade Federal de São Paulo - UNIFESP - São Paulo (SP) - Brasil ; Professora Titular da Universidade de Santo Amaro - UNIS A - São Paulo (SP), Brasil.

${ }^{3}$ Livre Docente em Informática Médica e Doutora em Enfermagem da Universidade Federal de São Paulo - UNIFESP - São Paulo (SP) - Brasil; Coordenadora do Núcleo de Informática em Enfermagem - NIEn/ UNIFESP.

${ }^{4}$ Doutora Enfermagem. Professora Adjunta da Universidade Federal de São Paulo - UNIFESP - São Paulo (SP) - Brasil.
} 


\section{INTRODUÇÃO}

O Diabetes Mellitus (DM) acomete cerca de 7,6\% da população brasileira entre 30 e 69 anos de idade. No entanto, aproximadamente, $50 \%$ dos pacientes desconhecem o diagnóstico e $24 \%$, reconhecidamente portadores de DM, não fazem qualquer tipo de tratamento. As complicações crônicas do DM são as principais responsáveis pela morbimortalidade desses pacientes $^{(1-4)}$.

Dentre as complicações podem ser citadas o "pé diabético" ou "pé em risco", que se constitui em problema de saúde pública, em razão da freqüência com que ocorre e do alto custo do tratamento. $\mathrm{Na}$ maior parte dos casos evolui para amputação dos membros inferiores, e sabe-se que no período de três anos após uma amputação de membro inferior, a porcentagem de sobrevida é de $50 \%$ e no prazo de dez anos o índice de mortalidade é de 39 a 68\%. Entre as causas desses óbitos é devido à infecção hospitalar ${ }^{(5-8)}$.

Ainda sobre o pé diabético Gross ${ }^{(3)} \mathrm{e}^{\mathrm{L}}$ Levin O’Neal ${ }^{(8)}$ destacam que: portadores de diabetes com lesões graves nos pés constituem $51 \%$ dos pacientes internados em enfermarias de endocrinologia nos hospitais universitários, com períodos de internação que podem chegar a 90 dias. A grande maioria dos casos graves que necessitam de internação hospitalar origina-se de úlceras superficiais ou de lesões pré ulcerativas nos pés, com diminuição da sensibilidade por neuropatia diabética, associada a pequenos traumas, geralmente causados por calçados inadequados, dermatoses ou manipulações impróprias dos pés pelos pacientes ou por pessoas não habilitadas.

Assim, embora as úlceras associadas à doença vascular periférica constituam a menor parcela das complicações, requerem cuidados imediatos e especializados. A atuação da equipe multidisciplinar e a avaliação clínica podem prevenir o surgimento de lesões nos pés por meio de consulta médica e ou de enfermagem, utilizando instrumentos especializados e procurando classificar o grau de risco das lesões.

A avaliação clínica baseia-se no exame físico dos pés que inclui inspeção e palpação da pele (coloração e temperatura); observação da presença de unhas encravadas ou deformadas e a estrutura dos pés (deformidade dos dedos e do arco plantar); áreas de calosidades, rachaduras, fissuras, bolhas, úlceras e micoses; a palpação dos pulsos arteriais tibial posterior e pedioso (que podem estar ausentes em 10,0\% dos indivíduos normais) e a avaliação da sensibilidade protetora plantar empregando o monofilamento de Semmes Wertein de 10 gr e ou diapasão de 128 Hertz. Além disto, os calçados devem ser inspecionados, verificando pontos de atrito ou de pressão plantar excessiva, desgaste irregular e presença de corpos estranhos. Segundo estes procedi- mentos básicos, os pacientes diabéticos com risco de apresentarem úlceras, poderão ser facilmente identificados ${ }^{(2,3,6,9-13)}$.

Para que estes procedimentos básicos sejam realizados, é necessário investir em medidas de conscientização e educação dos profissionais, pacientes e familiares com o objetivo de prevenir ou atenuar as complicações, levando em consideração o estilo de vida, atitudes individuais, nível sócio-econômico e a capacidade física dos portadores de DM. Para que esta educação seja eficaz e efetiva, o enfermeiro necessita atualizar-se no âmbito teóricoprático, utilizando entre outros meios a internet, que se tornou um dos mais importantes veículos de transmissão de informações ${ }^{(14-16)}$.

O emprego dos computadores encontra-se disseminado em todas as áreas da atividade humana, e a área da saúde vem se destacando pela intensa utilização das tecnologias computacionais, sobretudo no âmbito educacional $^{(15)}$.

Com eles, o universo da aprendizagem torna-se amplo, à medida que cada vez mais e mais pessoas utilizam a internet para a educação, em razão da facilidade de acesso nas escolas, no trabalho e em locais públicos. Em 2001, o número de internautas no Brasil era de 14 milhões, e em 2006 será de 42,3 milhões. Estes dados demonstram o crescimento do emprego dos computadores de maneira significativa, tornando-os cada vez mais acessíveis a todas as classes sociais ${ }^{(17)}$.

A Internet é um importante recurso de informação em saúde para os consumidores; muitos e muitos pacientes utilizam-se da internet para aprender sobre diagnósticos, revisão de possíveis tratamentos, checar os medicamentos e encontrar outros tipos de informações de saúde tanto para eles mesmos como para seus familiares ${ }^{(18)}$.

Marin ${ }^{(19)}$ cita que "os recursos oriundos desta tecnologia são de grande valia para a enfermagem quando aplicados, tanto no gerenciamento dos serviços de atendimento à saúde, ou seja, hospitais, unidades básicas de saúde e afins, quanto no apoio ao profissional nas atividades de assistência ao paciente".

O ensino pela Web não veio acabar com o ensinopresença de um educador e sim, trabalhar em paralelo com ele para que, no futuro, seja possível a aplicação na íntegra do ensino a distância (e-learning), quebrando assim a resistência das pessoas a este tipo de aplicação, e criando uma cultura de uso deste tipo de ferramenta que tem como vantagens a flexibilidade em termos de horário, o desenvolvimento de habilidades críticas no mundo globalizado, autonomia para resolução de problemas e a aprendizagem solo em termos de estudo, pesquisa e coleta de informações ${ }^{(20)}$.

Diante dos aspectos apresentados, a proposta deste trabalho foi descrever o processo de criação de um web site educacional para Enfermeiros que disponibilize 
informações sobre a avaliação e cuidados preventivos dos pés em pacientes com diabetes mellitus, e avaliar o conteúdo das informações disponibilizadas no web site com especialistas na área de informática, diabetes mellitus e educação a distância.

\section{MÉTODOS}

A disponibilização de informações pela infra-estrutura da World Wide Web é o foco principal desta pesquisa. Este estudo é uma pesquisa aplicada, inserida na linha de pesquisa Informática, Tecnologia da Informação e Comunicação em Saúde e Enfermagem do Núcleo de Informática em Enfermagem - NIEn/UNIFESP. Baseou-se no desenvolvimento ativo de um web site com a finalidade de disponibilizar informações sobre complicações nos pés de pacientes com diabetes mellitus. O estudo divide-se em duas partes: a construção e a validação do web site.

Para a evolução do estudo, foi adotado o modelo geral de criação de web sites sugerido por Trochim ${ }^{(21)}$. Este modelo envolve quatro grandes fases que são: conceituação; desenvolvimento; implementação e avaliação. $\mathrm{Na}$ fase de conceituação, determina-se o público - alvo, o objetivo, a análise e a definição do conteúdo que será abordado. Esta fase é direcionada à organização e disposição do conteúdo. Este estudo foi direcionado a Enfermeiros que atuam na assistência ao paciente diabético em geral e, em especial, aqueles com lesões nos pés. Após a reunião dos artigos e materiais de interesse para a elaboração do web site, foi feita a definição do conteúdo que contemplaria os objetivos previamente definidos para que as informações providenciassem o conhecimento necessário, capaz de auxiliar na prevenção da patologia.

Considerando a problemática da qualidade das informações disponibilizadas na World Wide Web, foram adotadas as normas de conduta da Health On The Net Foundation $^{(22)}$. Estas normas referem-se a Autoridade, Complementaridade, Confidencialidade, Atribuição, Justificativas, Transparência na propriedade, Transparência do patrimônio e Honestidade da publicidade e da política editorial.

A fase de desenvolvimento diz respeito à criação e estruturação do Web site. Para a criação do web site, foi necessária a participação da autora em cursos sobre a linguagem marcada de hipertexto (HTML) e edição de páginas Web (Dreamweaver), além de conhecimentos gerais de informática. Para desenvolvimento do web site foram utilizados os seguintes recursos de informática: computador com processador Pentium 4 com $32 \mathrm{MB}$ de memória RAM (Randomic Acces Memory) e disco rígido de $20 \mathrm{~GB}$ de capacidade de armazenamento com gravador de CD; notebbok Advent 7001 (Toshiba), Pentium 4 com 64 MB de memória RAM (Randomic Acces
Memory) e disco rígido de 20 GB de capacidade de armazenamento.

Para a obtenção das imagens, foram utilizados um scanner de mesa com capacidade de resolução de 9600 dpi (pontos por polegada) Hewlett-Packard ${ }^{\circledR}$ modelo 2200C; uma máquina fotográfica Nikon ${ }^{\circledR}$ para a digitalização das imagens e uma impressora a jato de tinta da Hewlett-Packard modelo 680C.

O Web site foi desenvolvido em um computador com o sistema operacional Windows ME da Microsoft Corporation $^{\circledR}$. Para a criação do hiperdocumento, foi escolhido o software Word 2000 da Microsoft Corporation ${ }^{\circledR}$ pela sua facilidade e sua flexibilidade com o editor de página web adotado. Como editor de página da web, foi selecionado o software Dreamweaver MX da Macromedia ${ }^{\circledR}$, que é um editor visual profissional que cria e gerencia sites e páginas da web. Com este programa, é fácil criar páginas compatíveis com diversos navegadores e plataformas. O Dreamweaver $\mathrm{MX}^{\circledR}$ oferece ferramentas avançadas de desenho e layout, bem como facilita a utilização dos recursos do HTML dinâmico, como os comportamentos e camadas. Para o editor de imagem adotou-se o software Fireworks MX Macromedia ${ }^{\circledR}$, que tem como propriedade, dividir imagens e compactá-las para transferências muito rápidas pela internet, incorporar efeitos de rollover em textos, além de gerar JAVASCRIPT para carregamentos de imagens para ação rápida quando o mouse move-se sobre o menu popup. O aplicativo permite também a possibilidade de salvamento de arquivos no formato GIF e JPEG. Para o teste das páginas em ambiente Internet, foi selecionado o (navegador) software Internet Explorer 5.0 da Microsoft Corporation ${ }^{\circledR}$. Este navegador possui facilidade com o editor de páginas Web adotado.

A fase de implementação envolveu a publicação do Web site na rede mundial de computadores. Assim, são descritos os processos de registro de domínio, contratação dos serviços de um provedor comercial, publicação e a atualização das informações a serem disponibilizadas.

$\mathrm{Na}$ fase de avaliação, as operações e o conteúdo do web site são examinados por especialistas na área de informática em educação e diabetes mellitus. Foram convidados dez especialistas sendo eles: quatro com especialidade em informática e educação à distância, quatro com experiência em diabetes e dois com experiência em informática. Todos os especialistas possuíam experiência mínima de oito anos na área de atuação e foram selecionados de acordo com alguns critérios: experiência na área de diabetes mellitus e cuidados com os pés; experiência em informática em saúde e experiência em educação à distância.

A solicitação para a participação na avaliação foi feita 
por meio de carta convite, contendo esclarecimentos sobre as finalidades e objetivos do estudo. A etapa seguinte foi a entrega do CD-ROM e do questionário de avaliação, acompanhado do termo de consentimento livre e esclarecido, estabelecendo-se o prazo de 15 dias, considerando-se a disponibilidade de local e tempo de melhor conveniência de cada avaliador.

O período de avaliação transcorreu na primeira semana de junho de 2004 para os avaliadores que aceitaram participar do estudo. O questionário de avaliação foi adaptado dos instrumentos já existentes na literatura ${ }^{(23-27)} \mathrm{e}$ continha nove questões fechadas onde o avaliador concebia um dos conceitos (excelente, muito bom, bom, regular ou ruim) relacionado a: velocidade no carregamento das páginas; design; informações objetivas; facilidade de navegação; organização clara e lógica; fotos e figuras; conteúdo; escrita correta e links. $\mathrm{O}$ instrumento de avaliação também continha duas questões abertas, nas quais os avaliadores poderiam opinar e fazer sugestões ou comentários a respeito do web site.

\section{RESULTADOS E DISCUSSÃO}

Foram criadas 68 páginas contendo 100 imagens (fotos, figuras). O tempo total gasto na construção do site foi, aproximadamente, de seis meses. O tamanho total do arquivo é 2,59 megabytes (mb), sendo as das páginas de 394 kilobytes $(\mathrm{kb})$ e das fotos com 2,13 megabytes (mb).

$\mathrm{O}$ acesso ao web site deve ser feito pelo endereço: www.unifesp.br/denf/nien, na opção pesquisa. A página inicial (home), foi elaborada dentro de um frameset triplo que auxilia na navegação de todo o site constituído de três páginas:

1. topo.htm, contendo o Banner personalizado para o web site, englobando o logotipo da Universidade Federal de São Paulo (UNIFESP). Ao passar o cursor no logotipo da UNIFESP, o usuário fará conexão com a home page da Universidade Federal de São Paulo;

2. menu.htm, contendo nove opções de menu, que foram criados com o Shockwave flash (arquivos gerados pelo Flash em páginas HTML [Hypertext Markup Language]);

3. main.htm, que é o frame utilizado para o carregamento das páginas, contendo link para os princípios da qualidade ditados pelo HON CODE, contendo também o seu logotipo onde o usuário poderá acessá-lo.

$\mathrm{Na}$ definição do frameset, o conjunto destas três páginas, formou a página index. htm, ou seja, a página inicial do website. A partir da página inicial, o usuário tem acesso a todo o conteúdo do site por meio do frame lateral esquerdo, contendo nove opções de menu.

\section{1- Descrição dos principais conteúdos do web site \\ Na página inicial Index, no topo está contido o logotipo}

do website e o da UNIFESP. Por intermédio do frameset lateral esquerdo, está disponibilizado o menu, onde o usuário inicia o acesso ao conteúdo didático-científico do site. Nesta página, estão apresentadas um link para o Health on the Net Foundation, e um link para o Microsoft Outlook, quando o usuário clicar no nome da autora do web site. No frame lateral esquerdo, estão disponibilizadas nove opções de menu, assim distribuídas: $\mathrm{O}$ que é o web site; Diabetes mellitus; Pé diabético; Neuropatias; Doença Vascular; Infecções; Amputações; Enfermagem e o Mapa do site.

As opções de menu seguem uma ordem didática, visando auxiliar o entendimento das informações. Além disso, a criação das páginas exigiu preocupação da autora em disponibilizar informações objetivas e claras, promovendo o interesse do usuário em navegar pelo site. A este propósito Salvador ${ }^{(28)}$ destaca que as informações devem ser claras e objetivas, sem excesso de texto e as páginas não devem demorar em carregar, o que levaria o usuário a desistir da consulta.

Selecionando a opção O que é o web site no frameset lateral esquerdo, abre a página contendo informações sobre a autora, a finalidade do estudo, o público alvo, além de links para "colaboradores", "links interessantes", "glossário com termos técnicos" e "referências bibliográficas".

No link Pé diabético, o usuário poderá visualizar a descrição do conceito e a epidemiologia do pé diabético, indispensáveis para o entendimento do web site. O pé diabético é uma das mais graves e onerosas complicações do diabetes mellitus. Uma estratégia que inclua prevenção, educação dos pacientes e dos profissionais da saúde, tratamento multidisciplinar das úlceras nos pés e sua rígida monitoração pode reduzir as taxas de amputações entre 49 e $85 \% \%^{(13)}$.

$\mathrm{Na}$ opção enfermagem, o usuário encontra informações sobre a importância da educação, tanto do enfermeiro como do paciente. Apresenta links para educação do paciente; exame físico dos pés; exame de sensibilidade dos pés; diapasão; curativos e desbridamento. A seqüência dos itens foi elaborada a fim de permitir melhor compreensão do usuário.

\section{2 - A avaliação do Web Site}

Dez especialistas da área de diabetes mellitus, informática e educação avaliaram o web site. Todos julgaram o conteúdo programático e os recursos computacionais usados no web site. A validação por meio de especialistas é um recurso útil ao se considerar que em nosso país ainda não existem métodos ou sistemas oficiais que controlem ou revisem a qualidade da informação de saúde na World Wide Web ${ }^{(26)}$.

Após a análise dos questionários respondidos pelos especialistas, algumas sugestões feitas foram incorporadas 
ao site, assim como a revisão ortográfica, com a finalidade de melhorar sua qualidade. As sugestões feitas foram a seqüência do menu para facilitar o entendimento do assunto abordado que foi alterada; foram introduzidas as páginas: pé diabético; o que é o web site pé diabético; referências bibliográficas e glossário de termos técnicos. Em todas as páginas os ícones de voltar e próximo para facilitar a navegação do usuário foram introduzidos. Outras sugestões que não comprometiam o conteúdo do web site ou o seu entendimento, tais como: a inserção de submenus nas páginas; acréscimo de mais informações sobre curativos e a alteração do banner principal, serão feitas futuramente no sentido de ampliar as informações referentes ao assunto.

Após a fase de avaliação dos especialistas e das modificações realizadas e, estando o mesmo em perfeitas condições para a inserção na Internet, o web site foi disponibilizado no provedor da Universidade Federal de São Paulo (UNIFESP), no Departamento de Enfermagem - Núcleo de Informática em Enfermagem.

\section{CONCLUSÃO}

O web site intitulado "Pé Diabético", desenvolvido no programa de Pós-Graduação em Enfermagem da Universidade Federal de São Paulo, está disponível na Internet no endereço: www.unifesp.br/denf/nien, na opção pesquisa.

O web site foi avaliado por dez especialistas das áreas de informática, diabetes mellitus e educação à distância, nos quesitos de conteúdo, design, interface e manipulação, que o consideraram (90\%) "excelente", "muito bom" e "bom". Algumas alterações foram feitas no web site de acordo com as sugestões dos especialistas e outras serão realizadas futuramente, que não comprometem sua qualidade.

\section{CONSIDERAÇÕES FINAIS}

Este estudo pretende contribuir para a prática de enfermagem com pacientes com pé diabético. Sendo, assim, a pesquisadora propõe algumas ações futuras baseadas nesta pesquisa:

1. O conteúdo deste web site deverá ser atualizado periodicamente, sendo acrescentadas novas informações pertinentes ao assunto e ou substituídas às consideradas obsoletas.

2. Incluir um grupo de discussão virtual, no qual os enfermeiros e interessados no assunto possam trocar experiências e conteúdos a respeito do tema.

3. Despertar em outros enfermeiros o interesse para desenvolver web sites com novos temas, visando com isto, a formação de mais profissionais especialistas na área e o aperfeiçoamento do conhecimento em tecnologia da informação em enfermagem.
O avanço tecnológico, a globalização e a mudança exigida das pessoas no ambiente de trabalho determinam uma nova postura profissional do enfermeiro. Mais do que conhecimento, ele precisa de atualização constante, por meio da sua educação permanente e a informática é uma ferramenta imprescindível para facilitar esta aprendizagem em qualquer ambiente, no trabalho ou fora dele. Sendo assim, é imprescindível que o enfermeiro domine esta área de conhecimento e a aprimore, contribuindo para a divulgação cientifica de praticas inovadoras para a enfermagem.

\section{REFERÊNCIAS}

1. Brasil. Ministério da Saúde. Estudo multicêntrico sobre a prevalência do diabetes mellitus no Brasil. Brasilia: Ministério da Saúde ; 1991.

2. James H. Foot problems in diabetes. Nurs Times. 1995; 91(39): 65-6, 69-70, 72 .

3. Gross JL. Detecção e tratamento das complicações crônicas do Diabetes Mellitus. Consenso Brasileiro. Arq Bras Endocrinol Metab. 1999; 43(1):7-14.

4. Brasil. Ministério da Saúde. Secretaria de políticas de saúde. Manual de hipertensão arterial e diabetes mellitus. Brasília: Ministério da Saúde; 2002.

5. Gamba MA. Amputações por diabetes mellitus, uma prática prevenível? Acta Paul Enferm. 1998; 11(3): 92-100.

6. Armstrong DG, Lavery LA. Diabetic foot ulcers: prevention, diagnosis and classification. Am Fam Physician. 1998; 57 (6):1325-32; 1337-8. Review.

7. Lellis VLC. Avaliação, prevenção e intervenção no “pé em risco". Diabetes Clínica.2000; 4(5): 371-75.

8. Levin \& O’Neal. O Pé Diabético. Rio de Janeiro:Di livros; 2001.

9. Pérez-Pasten E. Manual para el paciente con diabetes mellitus. México: Editorial Soluciones Gráficas; 1997.

10. American Diabetes Association. Atualização. Diabetes Clínica. 2000; 4:102-132.

11. Pedrosa HC, Nery ES, Sena FV, et al. O desafio do projeto Salvando o Pé Diabético - Terapêutica em Diabetes. Boletim do Centro BD. 1998; 19: 1-10.

.12. Pedrosa H. Pés: cuide bem deles. Jornal Smithkline Beecham. 2000; 4:4-5.

13. Grupo de Trabalho Internacional sobre Pé Diabético. Consenso Internacional sobre Pé Diabético. Brasília: Secretaria de Estado de Saúde do Distrito Federal; 2001.

14. Block DE, Pollock TJ, Hutton SJ. Technoglitches in distance education. Comput Nurs. 1999; 17 (5): 2324.

15. Mills AC. Creating Web-based, multimedia and interactive courses for distance learning. Comput Nurs. 2000; 18(3):125-31. 
16. Soon KH, Sook KI, Jung CW, Im KM. The effects of Internet-based distance learning in nursing. Comput Nurs. 2000; 18(1):19-25.

17. Computerworld. A internet e a educação 2002 [texto na Internet]. [citado 2003 mar 15]. Disponível em: http:// www.computerworld.com.br

18. Oermann MH, Pasma J. Evaluation by consumers of quality care information on the internet. J Nurs Care Qual. 2001; 15(3):50-8.

19. Marin HF. Informática em enfermagem. São Paulo: EPU; 1995.

20. Belloni ML. Educação à distância. São Paulo: Autores Associados; 1999.

21. Trochim WMK. Evaluating Web Sites. Cornell University; 1999 [texto on the Internet]. [cited 2002 Mar 3]. Available from: http:// trochim.human.cornell.edu/webevol/webintro/ webintro.htm (03 mar. 2002).

22. Health on the Net Foundation [sítio na Internet]. Código de conduta (HON) para sites Web de medicina e saúde; 2002. [citado 2003 Mar 4]. Disponível em: http:// www.hon.ch/HONcode/portuguese. [Tradução para o português de: César de Azevedo Gil - Banco Nacional de Desenvolvimento Econômico e Social BNDES, February 2; 2000].
23. Mitushima SM. Desenvolvimento de um web site educacional sobre monitorização hemodinâmica: o uso do cateter de Swan-Ganz [tese]. São Paulo: Escola Paulista de Medicina. Enfermagem.Universidade Federal de São Paulo; 2004.

24. Nogueira VO. Informações on-line sobre transporte intra-hospitalar de pacientes críticos adultos [tese]. São Paulo: Escola Paulista de Medicina. Enfermagem. Universidade Federal de São Paulo; 2003.

25. Diogo RCS. Desenvolvimento do web site educacional sobre intervenção de enfermagem: aspiração de secreções traqueobrônquicas [tese]. São Paulo: Escola Paulista de Medicina. Enfermagem. Universidade Federal de São Paulo; 2001.

26. Marques IR. Enfermagem na Web: o processo de criação e validação de um Web site sobre doença coronariana [tese]. São Paulo: Escola Paulista de Medicina. Universidade Federal de São Paulo; 2000.

27. Motta MCS. Software educacional de enfermagem em puericultura: desenvolvimento e validação [tese]. Rio de Janeiro: Escola de Enfermagem Anna Nery da Universidade Federal do Rio de Janeiro; 2000.

38. Salvador B. Multimídia e Internet [texto na Internet]. 2000 [citado 2003 Mar 4]. Disponível em: http:// www.virtual.epm.br/material/tis 